\title{
THE DE BRANGES-ROVNYAK MODEL WITH FINITE-DIMENSIONAL COEFFICIENTS
}

\author{
JAMES GUYKER
}

\begin{abstract}
A characterization in terms of the canonical model spaces of $\mathrm{L}$. de Branges and J. Rovnyak is obtained for Hilbert spaces of formal power series with vector coefficients which satisfy a difference-quotient inequality, thereby extending the closed ideal theorems of A. Beurling and P. D. Lax.
\end{abstract}

\section{INTRODUCTION}

This paper extends the well-known invariant subspace characterization of A. Beurling [3] and P. D. Lax [11] for the shift on the Hardy space of square summable power series with vector coefficients (cf. [10, 13-15]). The focus is instead on certain (not necessarily orthogonal) complements of contractively contained invariant manifolds of the shift. These are the spaces $\mathscr{H}(B)$ of L. de Branges and J. Rovnyak [6-8]. In the Beurling-Lax theory, the key point is a dimension inequality. The inequality is trivial when the coefficient space has infinite dimension, so the essential content is in the finite-dimensional case. Previously only special cases of the more abstract problem have been treated $[6,9]$, but our methods generalize an argument from [7, Theorem 6]. The main difficulty again comes down to a dimension inequality in the finite-dimensional case. The purpose here is to derive new results on the structure of $\mathscr{H}(B)$ spaces which reveal what is needed for the inequality to hold. As a consequence, we obtain a complete characterization of the spaces $\mathscr{H}(B)$.

\section{2. $\mathscr{H}(B)$ SPACES}

A basic concept in the de Branges-Rovnyak theory is complementation: A Hilbert space $\mathscr{F}$ is contained contractively in a Hilbert space $\mathscr{K}$ if $\mathscr{F}$ is a submanifold of $\mathscr{K}$ and if the inclusion map of $\mathscr{F}$ into $\mathscr{K}$ is a contraction. If $\mathscr{F}$ is contained contractively in $\mathscr{K}$, then the space complementary to $\mathscr{F}$ in $\mathscr{K}$ is the Hilbert space $\mathscr{G}$ of elements $g$ of $\mathscr{K}$ with the property that

$$
\|g\|_{\mathscr{G}}^{2}=\sup \left\{\|g+f\|_{\mathscr{K}}^{2}-\|f\|_{\mathscr{F}}^{2}: f \in \mathscr{F}\right\}
$$

is finite. The space $\mathscr{G}$ is contained contractively in $\mathscr{K}$. Moreover, $\mathscr{G}$ is the unique Hilbert space such that the inequality $\|k\|_{\mathscr{K}}^{2} \leq\|f\|_{\mathscr{g}}^{2}+\|g\|_{\mathscr{G}}^{2}$ holds whenever $k=f+g$ is a decomposition of $k$ in $\mathscr{K}$ into $f$ in $\mathscr{F}$ and $g$ in

Received by the editors May 6, 1993 and, in revised form, January 3, 1994; orginally communicated to the Proceedings of the AMS by Palle E. T. Jorgensen.

1991 Mathematics $\mathbf{r}^{\prime}$ ibject Classification. Primary 46E22, 47A45. 
$\mathscr{G}$ and such that every element $k$ in $\mathscr{K}$ admits a decomposition for which equality holds.

Let $\mathscr{C}$ be a finite-dimensional Hilbert space, and let $\mathscr{H}$ be a Hilbert space of formal power series $f(z)$ whose coefficients are in $\mathscr{C}$ such that

$$
\|[f(z)-f(0)] / z\|_{\mathscr{H}}^{2} \leq\|f(z)\|_{\mathscr{P}}^{2}-|f(0)|_{\mathscr{C}}^{2} .
$$

Then $\mathscr{H}$ is contained contractively in $\mathscr{C}(z)$, the Hilbert space of square summable power series $\sum a_{n} z^{n}$ with $a_{n}$ in $\mathscr{C}$ and norm given by $\left\|\sum a_{n} z^{n}\right\|_{\mathscr{C}(z)}^{2}=$ $\sum\left|a_{n}\right|_{\mathscr{C}}^{2}$.

Let $B(z)$ be a power series whose coefficients are operators on $\mathscr{C}$ such that $\|B(z) f(z)\|_{\mathscr{C}(z)} \leq\|f(z)\|_{\mathscr{C}(z)}$ whenever $f(z)$ is in $\mathscr{C}(z)$. Cauchy multiplication by $B(z)$ thus defines a contraction operator on $\mathscr{C}(z)$ which will be denoted by $T_{B}$. The range $\mathscr{M}(B)$ of $T_{B}$ becomes a Hilbert space in the unique norm with the property that $\left\|T_{B} f\right\|_{\mathscr{M}(B)}=\|f\|_{\mathscr{C}(z)}$ whenever $f$ is orthogonal to the kernel of $T_{B}$. Furthermore, $\mathscr{M}(B)$ is contained contractively in $\mathscr{C}(z)$, and multiplication by $z$ is a contraction on $\mathscr{M}(B)$.

The de Branges-Rovnyak space $\mathscr{H}(B)$ is defined to be the complementary space to $\mathscr{M}(B)$ in $\mathscr{C}(z)$. The space $\mathscr{H}(B)$ satisfies (1) and is an underlying space for canonical models of contractions on Hilbert space $[1,2,12,16,17]$.

Multiplication by $z$ is a contraction on the space $\mathscr{M}$ complementary to $\mathscr{H}$ in $\mathscr{C}(z)$. In [6] (cf. [5, Theorem 6]), de Branges extended the Beurling-Lax theorem by showing that if multiplication by $z$ is isometric on $\mathscr{M}$, then $\mathscr{H}$ is isometrically equal to a space $\mathscr{H}(B)$. It should be further noted that when $\mathscr{C}$ is infinite dimensional, any space $\mathscr{H}$ which satisfies $(1)$ is isometrically equal to a space $\mathscr{H}(B)[4$, Theorem 11].

Let $\mathscr{H}(B)$ be a given space. Then $\mathscr{H}(B)$ is also contained contractively in $\mathscr{H}(z B)$. The space $\mathscr{H}(z B)$ may be obtained as those elements $h(z)$ of $\mathscr{C}(z)$ such that $[h(z)-h(0)] / z$ is in $\mathscr{H}(B)$ and $\|h(z)\|_{\mathscr{H}(z B)}^{2}=\|[h(z)-h(0)] / z\|_{\mathscr{H}(B)}^{2}+$ $|h(0)|_{\mathscr{C}}^{2}$. The complementary space to $\mathscr{H}(B)$ in $\mathscr{H}(z B)$ is the space $B(z) \mathscr{C}$ with $\|B(z) c\|_{B(z) \mathscr{C}}=|c|_{\mathscr{C}}$ for every $c$ orthogonal to $\mathscr{C} \cap \operatorname{ker} T_{B}$. Let us define linear transformations $J_{ \pm}$from $\mathscr{H}(B)$ into $\mathscr{C}$, with ranges denoted $\mathscr{C}_{ \pm}$, as follows: $J_{+} f=f(0)$ and $J_{-}$is the operator whose adjoint is given by $J_{-}^{*} c=$ $[B(z)-B(0)] c / z$. Let $B(z)=\sum B_{n} z^{n}$, and let $\bar{B}_{n}$ be the adjoint of $B_{n}$ on $\mathscr{C}$. Then $J_{+}^{*} c=[1-B(z) \bar{B}(0)] c$; and since $\mathscr{C}$ is finite dimensional, $\mathscr{C}_{+}=\left(1-B_{0} \bar{B}_{0}\right) \mathscr{C}$ and $\mathscr{C}_{-}=\left(\bigvee_{n \geq 1} \bar{B}_{n} \mathscr{C}\right) \subseteq\left(1-\bar{B}_{0} B_{0}\right) \mathscr{C}$.

Let $R(0)$ denote the difference-quotient transformation on $\mathscr{H}(B)$, which maps $f(z)$ into $[f(z)-f(0)] / z$. Then $R(0)^{*} f(z)=z f(z)-B(z) J_{-} f$ so that $\left[1-R(0) R(0)^{*}\right] f(z)=[B(z)-B(0)]\left(J_{-} f\right) / z$ and $\left[1-R(0)^{*} R(0)\right] f(z)=$ $\left(J_{+} f\right)+B(z) J_{-} R(0) f$. Note that if $\left[1-R(0)^{*} R(0)\right] f(z)=c+B(z) c_{-}$with $c$ in $\mathscr{C}$ and $c_{-}$in $\mathscr{C}_{-}$, then necessarily $c=J_{+} f$ and $c_{-}=J_{-} R(0) f$. Therefore, since $\operatorname{dim} \mathscr{C}$ is finite,

$$
\begin{aligned}
\operatorname{rank}\left[1-R(0)^{*} R(0)\right] & =\operatorname{dim}\left\{\left(J_{+} f, J_{-} R(0) f\right): f \in \mathscr{H}(B)\right\} \\
& \geq \operatorname{dim} \mathscr{C}_{+}=\operatorname{rank}\left(1-\bar{B}_{0} B_{0}\right) \\
& \geq \operatorname{dim} \mathscr{C}_{-}=\operatorname{rank}\left[1-R(0) R(0)^{*}\right] .
\end{aligned}
$$

More precisely, the following will turn out to be a defining property of the spaces $\mathscr{H}(B)$. 
Theorem 1. Let $R(0)$ be the difference-quotient transformation on a given space $\mathscr{H}(B)$. Then

$$
\operatorname{rank}\left[1-R(0)^{*} R(0)\right]=\operatorname{dim}\{c \in \mathscr{C}: B(z) c \in \mathscr{H}(B)\}+\operatorname{rank}\left[1-R(0) R(0)^{*}\right] .
$$

Proof. Suppose that $B(z) c$ is in $\mathscr{H}(B)$. Then $c=\left(J_{-} f\right)+d$ where $f$ is in $\mathscr{H}(B)$ and $[B(z)-B(0)] d / z=0$. Moreover,

$$
\left[1-R(0)^{*} R(0)\right]\left\{\left[R(0)^{*} f\right]+B(z) c\right\}=\left(B_{0} d\right)+B(z) J_{-} f .
$$

Let $J_{-} f_{1}, \ldots, J_{-} f_{s_{0}}$ be a basis for the subspace $\mathscr{C}_{-}^{\prime}=\left\{c \in \mathscr{C}_{-}: B(z) c \in\right.$ $\mathscr{H}(B)\}$, and let $J_{+} g_{1}, \ldots, J_{+} g_{t}$ be a basis for $\mathscr{C}_{+}$where $f_{i}$ and $g_{j}$ are in $\mathscr{H}(B)$ for all $i$ and $j$. Suppose that there are constants $\lambda_{1}, \ldots, \lambda_{s_{0}+t}$ such that

$$
\begin{aligned}
0= & \sum_{1}^{s_{0}} \lambda_{i}\left[1-R(0)^{*} R(0)\right]\left\{\left[R(0)^{*} f_{i}\right]+B(z) J_{-} f_{i}\right\} \\
& +\sum_{1}^{t} \lambda_{s_{0}+j}\left[1-R(0)^{*} R(0)\right] g_{j} .
\end{aligned}
$$

Equivalently by (4) we have

$$
0=\left(\sum_{1}^{t} \lambda_{s_{0}+j} J_{+} g_{j}\right)+B(z) J_{-}\left[\sum_{1}^{t} \lambda_{s_{0}+j} R(0) g_{j}+\sum_{1}^{s_{0}} \lambda_{i} f_{i}\right]
$$

so that $\sum \lambda_{s_{0}+j} J_{+} g_{j}=0$ and hence $\lambda_{s_{0}+j}=0 \quad(j=1, \ldots, t)$. It follows that $\sum \lambda_{i} J_{-} f_{i}=0$ and thus $\lambda_{i}=0$ for all $i$. Therefore,

$$
\operatorname{rank}\left[1-R(0)^{*} R(0)\right] \geq s_{0}+t .
$$

Let $c_{i}=J_{-} f_{i} \quad\left(i=1, \ldots, s_{0}\right)$ and expand $\left\{c_{i}\right\}$ to a basis $c_{1}, \ldots, c_{s}$ of $\{c \in \mathscr{C}: B(z) c \in \mathscr{H}(B)\}$. For every $j>s_{0}$ let us write $c_{j}=\left(J_{-} f_{j}\right)+d_{j}$ as above where $f_{j}$ is in $\mathscr{H}(B)$ and $d_{j}$ is orthogonal to $\mathscr{C}_{-}$. By (4), $B_{0} d_{j}$ is in $\mathscr{C}_{+}$, so it is in $\left(B_{0} \mathscr{C}\right) \cap\left(1-B_{0} \bar{B}_{0}\right) \mathscr{C}$. But since $\mathscr{C}$ is finite dimensional, it follows that this intersection coincides with $B_{0}\left(1-\bar{B}_{0} B_{0}\right) \mathscr{C}$, and hence $B_{0} d_{j}=B_{0} e_{j}$ where $e_{j}$ is in $\left(1-\bar{B}_{0} B_{0}\right) \mathscr{E}$. Thus $d_{j}-e_{j}$ is in $\operatorname{ker} B_{0}$, which is also contained in $\left(1-\bar{B}_{0} B_{0}\right) \mathscr{C}$, and consequently $d_{j}$ is in $\left[\left(1-\bar{B}_{0} B_{0}\right) \mathscr{C}\right] \ominus \mathscr{C}_{-}$.

Now $\left\{d_{j}: j>s_{0}\right\}$ is linearly independent: For suppose $\sum \alpha_{j} d_{j}=0$. Then $\sum_{j>s_{0}} \alpha_{j} c_{j}=\sum_{j>s_{0}} \alpha_{j} J_{-} f_{j}$ is in $\mathscr{C}_{-}^{\prime}$, so there exist $\beta_{i}$ such that $\sum_{j>s_{0}} \alpha_{j} c_{j}=$ $\sum_{i \leq s_{0}} \beta_{i} c_{i}$. Since $\left\{c_{i}\right\}$ is linearly independent, $\alpha_{j}=0$ for all $j$, and hence

$$
\begin{aligned}
t & =\operatorname{dim} \mathscr{C}_{+}=\operatorname{rank}\left(1-B_{0} \bar{B}_{0}\right)=\operatorname{rank}\left(1-\bar{B}_{0} B_{0}\right) \\
& =\operatorname{dim}\left\{\left[\left(1-\bar{B}_{0} B_{0}\right) \mathscr{C}\right] \ominus \mathscr{C}_{-}\right\}+\operatorname{dim} \mathscr{C}_{-} \\
& \geq\left(s-s_{0}\right)+\operatorname{rank}\left[1-R(0) R(0)^{*}\right] .
\end{aligned}
$$

In conjunction with (5) we have

$$
\operatorname{rank}\left[1-R(0)^{*} R(0)\right] \geq s+\operatorname{rank}\left[1-R(0) R(0)^{*}\right] .
$$

To verify the reverse inequality, it suffices to show that there exist $r=$ $\operatorname{rank}\left[1-R(0)^{*} R(0)\right]-\operatorname{rank}\left[1-R(0) R(0)^{*}\right]$ linearly independent vectors $a_{i}$ in $\mathscr{C}$ such that $B(z) a_{i}$ is in $\mathscr{H}(B)$. By inequalities (2) and (3), it follows that $r=r_{0}+r_{1}$ where $r_{0}=\operatorname{rank}\left[1-R(0)^{*} R(0)\right]-\operatorname{dim} \mathscr{C}_{+}$and $r_{1}=$ $\operatorname{dim}\left\{\left[\operatorname{ran}\left(1-\bar{B}_{0} B_{0}\right)\right] \ominus \mathscr{C}_{-}\right\}$. 
Suppose that $r_{0}>0$ and recall the basis $\left\{J_{+} g_{j}\right\}$ of $\mathscr{C}_{+}$. As above, $\{[1-$ $\left.\left.R(0)^{*} R(0)\right] g_{j}\right\}$ is linearly independent, so if $\mathscr{G}$ is its span, then there are $r_{0}$ vectors $\left[1-R(0)^{*} R(0)\right] \hat{g}_{i} \quad\left(i=1, \ldots, r_{0}\right)$, with $\hat{g}_{i}$ in $\mathscr{H}(B)$, which form a basis of $\operatorname{ran}\left[1-R(0)^{*} R(0)\right] \ominus \mathscr{G}$. Now there exist constants $\lambda_{i j}$ such that $J_{+} \hat{g}_{i}=\sum_{j=1}^{t} \lambda_{i j} J_{+} g_{j}$ for each $i$. Let us define $a_{i}=J_{-} R(0)\left(\hat{g}_{i}-\sum_{j} \lambda_{i j} g_{j}\right)$ for $i=1, \ldots, r_{0}$. Then $B(z) a_{i}=\left[1-R(0)^{*} R(0)\right]\left(\hat{g}_{i}-\sum_{j} \lambda_{i j} g_{j}\right)$ is in $\mathscr{H}(B)$, and $\left\{a_{1}, \ldots, a_{r_{0}}\right\}$ is linearly independent: Suppose that $\sum \mu_{i} a_{i}=0$. Then

$$
\sum \mu_{i}\left[1-R(0)^{*} R(0)\right] \hat{g}_{i}=\sum_{i} \mu_{i}\left[1-R(0)^{*} R(0)\right]\left(\sum_{j} \lambda_{i j} g_{j}\right)
$$

which must be zero since it is in both $\mathscr{G}$ and $\mathscr{G}^{\perp}$. Therefore $\mu_{i}=0$ for every $i$.

Next, suppose that $r_{1}>0$ and let $\hat{d}_{1}, \ldots, \hat{d}_{r_{1}}$ be a basis of $\left[\operatorname{ran}\left(1-\bar{B}_{0} B_{0}\right)\right] \ominus$ $\mathscr{C}_{-}$. Then $B(z) \hat{d}_{j}=B_{0} \hat{d}_{j}$ and $\hat{d}_{j}=\left(1-\bar{B}_{0} B_{0}\right) b_{j}$ for some $b_{j}$ in $\mathscr{C}$. Let $\hat{f}_{j}(z)=[1-B(z) \bar{B}(0)] B_{0} b_{j}$ and define $a_{r_{0}+j}=\hat{d}_{j}+J_{-} R(0) \hat{f}_{j}$ for $j=1, \ldots, r_{1}$. Then $B(z) a_{r_{0}+j}=\left[1-R(0)^{*} R(0)\right] \hat{f}_{j}$ is in $\mathscr{H}(B)$.

Finally, $\left\{a_{i}: i=1, \ldots, r=r_{0}+r_{1}\right\}$ is linearly independent: Suppose that there are constants $\nu_{1}, \ldots, \nu_{r}$ such that

$$
0=\sum \nu_{i} a_{i}=\sum_{1}^{r_{0}} \nu_{i} a_{i}+\sum_{1}^{r_{1}} \nu_{r_{0}+j}\left[\hat{d}_{j}+J_{-} R(0) \hat{f}_{j}\right] .
$$

It follows that $\sum_{1}^{r_{1}} \nu_{r_{0}+j} \hat{d}_{j}=0$ since $a_{i} \quad\left(1 \leq i \leq r_{0}\right)$ and $J_{-} R(0) \hat{f}_{j} \quad(1 \leq j \leq$ $\left.r_{1}\right)$ are in $\mathscr{C}_{-}$, and $\hat{d}_{j}$ is orthogonal to $\mathscr{C}_{-}$for every $j$. Therefore $\nu_{r_{0}+j}=0$ $\left(j=1, \ldots, r_{1}\right)$, and consequently $\sum_{1}^{r_{0}} \nu_{i} a_{i}=0$ so that $\nu_{i}=0$ for all $i$.

\section{THE CHARACTERIZATION}

Let $\mathscr{H}$ be a space which satisfies (1), and let $\mathscr{H}^{\prime}$ be the Hilbert space of all power series $h(z)$ such that $[h(z)-h(0)] / z$ is in $\mathscr{H}$ with $\|h(z)\|_{\mathscr{P}^{\prime}}^{2}=$ $\|[h(z)-h(0)] / z\|_{\mathscr{H}}^{2}+|h(0)|_{\mathscr{C}}^{2}$. Then $\mathscr{H}^{\prime}$ satisfies $(1)$, and $\mathscr{H}$ is contained contractively in $\mathscr{H}^{\prime}$. Let $\mathscr{R}$ be the complementary space to $\mathscr{H}$ in $\mathscr{H}^{\prime}$, and let $i_{\mathscr{H}}$ and $i_{\mathscr{R}}$ denote the respective inclusion maps of $\mathscr{l}$ and $\mathscr{R}$ into $\mathscr{H}$ '. Then every $h$ in $\mathscr{H}^{\prime}$ admits the unique decomposition $h=\left(i_{\mathscr{C}}^{*} h\right)+\left(i_{\mathscr{R}}^{*} h\right)$ where $\|h\|_{\mathscr{R}}^{2}=\left\|i_{\mathscr{H}}^{*} h\right\|_{\mathscr{H}}^{2}+\left\|i_{\mathscr{R}}^{*} h\right\|_{\mathscr{R}}^{2}$.

A fundamental result from the theory of $\mathscr{H}(B)$ spaces is: $\mathscr{H}$ is isometrically equal to a space $\mathscr{H}(B)$ if and only if the dimension of $\mathscr{R}$ does not exceed the dimension of $\mathscr{C}$ [6]. More generally, if $\mathscr{C} \subseteq \tilde{\mathscr{C}}$ and $\operatorname{dim} \mathscr{R} \leq \operatorname{dim} \tilde{\mathscr{C}}$, then $\mathscr{H}$ is a space $\mathscr{H}(\tilde{B})$ where the coefficients of $\tilde{B}(z)$ act on $\tilde{\mathscr{C}}$.

Lemma. Let $\mathscr{F}$ be the subspace of elements of $\mathscr{H}$ for which equality holds in (1). Then $\mathscr{R}$ and $\mathscr{H} \cap \mathscr{R}$ are contained in $\mathscr{H}^{\prime} \ominus \mathscr{F}$ and $\mathscr{H} \ominus \mathscr{F}$ respectively. Moreover, $\operatorname{dim} \mathscr{R}=\operatorname{dim} \mathscr{H}^{\prime} \ominus \mathscr{F}$ and $\operatorname{dim} \mathscr{H} \cap \mathscr{R}=\operatorname{dim} \mathscr{H} \ominus \mathscr{F}$.

Proof. As in [9], $\mathscr{F}$ is a (closed) subspace of $\mathscr{H}$ and is contained isometrically in $\mathscr{H}^{\prime}$. Therefore for any $f$ in $\mathscr{F}$ and $g$ in $\mathscr{R}$, we have

$$
\langle f, g\rangle_{\mathscr{C}^{\prime}}=\left\langle f, i_{\mathscr{R}} g\right\rangle_{\mathscr{C}}=\left\langle i_{\mathscr{R}}^{*} f, g\right\rangle_{\mathscr{R}}=\langle 0, g\rangle_{\mathscr{R}}=0 \text {. }
$$

Hence $\mathscr{R}$ is a subset of $\mathscr{H}^{\prime} \ominus \mathscr{F}$. 
The restriction of $i_{\mathscr{R}}^{*}$ to $\mathscr{H}^{\prime} \ominus \mathscr{F}$ is linear and continuous and has trivial kernel: if $i_{\mathscr{R}}^{*} h=0$ for some $h$ in $\mathscr{H}^{\prime} \ominus \mathscr{F}$, then $i_{\mathscr{H}}^{*} h=h$, so $h$ is also in $\mathscr{F}$, and thus $h=0$. It follows that $\operatorname{dim} \mathscr{H}^{\prime} \ominus \mathscr{F}=\operatorname{dim} i_{\mathscr{R}}^{*}\left(\mathscr{H}^{\prime} \ominus \mathscr{F}\right) \leq \operatorname{dim} \mathscr{R}$, and hence $\operatorname{dim} \mathscr{R}=\operatorname{dim} \mathscr{H}^{\prime} \ominus \mathscr{F}$.

Next, let $g$ be in $\mathscr{H} \cap \mathscr{R}$. Then $g$ is in $\mathscr{L}^{\prime} \ominus \mathscr{F}$ but also in $\mathscr{H} \ominus \mathscr{F}$ since for any $f$ in $\mathscr{F}$

$$
\langle f, g\rangle_{\mathscr{H}}=\left\langle i_{\mathscr{H}}^{*} f, g\right\rangle_{\mathscr{H}}=\left\langle f, i_{\mathscr{L}} g\right\rangle_{\mathscr{H}^{\prime}}=\langle f, g\rangle_{\mathscr{H}^{\prime}}=0 .
$$

Therefore $(\mathscr{H} \cap \mathscr{R}) \subseteq(\mathscr{H} \ominus \mathscr{F})$. Finally $\operatorname{dim} \mathscr{H} \cap \mathscr{R}=\operatorname{dim} \mathscr{H} \ominus \mathscr{F}$ as above since $i_{\mathscr{R}}^{*}(\mathscr{H} \ominus \mathscr{F})$ is contained in $\mathscr{H} \cap \mathscr{R}$.

The following will distinguish the spaces $\mathscr{H}(B)$.

Corollary 1. Let $\mathscr{F}(B)$ be the subspace of elements of a given space $\mathscr{H}(B)$ for which equality holds in (1). Then

$$
\operatorname{dim} J_{+} \mathscr{F}(B)=\operatorname{dim}\left(\mathscr{C} \cap \operatorname{ker} T_{B}\right)+\operatorname{rank}\left[1-R(0) R(0)^{*}\right] .
$$

Proof. Since $B(z) \mathscr{C}$ is finite dimensional, the lemma implies that $\mathscr{H}(B) \ominus$ $\mathscr{F}(B)$ coincides with $\mathscr{H}(B) \cap B(z) \mathscr{C}$. By (1), the kernel of $1-R(0)^{*} R(0)$ is contained in $\mathscr{F}(B)$ and is exactly the kernel of the restriction of $J_{+}$to $\mathscr{F}(B)$. Thus since $1-R(0)^{*} R(0)$ has finite rank and

$$
J_{+} \mathscr{F}(B)=J_{+}\left\{\operatorname{ran}\left[1-R(0)^{*} R(0)\right] \cap \mathscr{F}(B)\right\},
$$

it follows that

$$
\begin{aligned}
\operatorname{rank}\left[1-R(0)^{*} R(0)\right]= & \operatorname{dim}\left\{\operatorname{ran}\left[1-R(0)^{*} R(0)\right] \cap \mathscr{F}(B)\right\} \\
& +\operatorname{dim}[\mathscr{H}(B) \ominus \mathscr{F}(B)] \\
= & \operatorname{dim} J_{+} \mathscr{F}(B)+\operatorname{dim}[\mathscr{H}(B) \cap B(z) \mathscr{C}] .
\end{aligned}
$$

The corollary now follows from Theorem 1 since we also have

$$
\begin{aligned}
\operatorname{rank}\left[1-R(0)^{*} R(0)\right]= & \operatorname{dim}\left(\mathscr{C} \cap \operatorname{ker} T_{B}\right)+\operatorname{dim}[\mathscr{H}(B) \cap B(z) \mathscr{C}] \\
& +\operatorname{rank}\left[1-R(0) R(0)^{*}\right] .
\end{aligned}
$$

By [7, Lemma 4], equality holds in (1) for a given space $\mathscr{H}(B)$ if and only if $\mathscr{H}(B)$ contains no nonzero element of the form $B(z) c$ with $c$ in $\mathscr{C}$. An immediate consequence of the above results is

Corollary 2. Let $\mathscr{H}(B)$ be a given space. Then $\operatorname{rank}\left[1-R(0)^{*} R(0)\right]=$ rank $\left[1-R(0) R(0)^{*}\right]$ if and only if equality holds in (1) for every $f(z)$ and there is no nonzero vector $c$ such that $B(z) c=0$.

We now have the proposed characterization.

Theorem 2. Let $\mathscr{H}$ be a Hilbert space of formal power series which satisfies (1), and let $\mathscr{F}$ be the subspace of those series for which equality holds in (1). Then $\mathscr{H}$ is isometrically equal to a space $\mathscr{H}(B)$ if and only if the dimension of the space of constant coefficients of elements of $\mathscr{F}$ is at least the rank of $1-T T^{*}$ where $T$ is the difference-quotient transformation on $\mathscr{H}$.

Proof. Any space $\mathscr{H}(B)$ has the stated property by Corollary 1 .

Conversely, suppose that $\mathscr{H}$ is a space which satisfies (1) and the dimension hypothesis. Let $\mathscr{H}^{\prime}, \mathscr{R}, i_{\mathscr{R}}$ and $i_{\mathscr{R}}$ be defined as above, and let $f(z)$ and $g(z)$ be in $\mathscr{H}$. Since 


$$
\left\langle i_{\mathscr{H}}^{*} z f(z), g(z)\right\rangle_{\mathscr{H}}=\left\langle z f(z), i_{\mathscr{H}} g(z)\right\rangle_{\mathscr{H}^{\prime}}=\langle f(z), T g(z)\rangle_{\mathscr{H}},
$$

it follows that $T^{*} f(z)=i_{\mathscr{H}}^{*} z f(z)$.

Let $S$ denote the difference-quotient transformation on $\mathscr{H}^{\prime}$. Then

$$
\begin{aligned}
\left(1-T T^{*}\right) f(z) & =f(z)-T i_{\mathscr{H}}^{*} z f(z) \\
& =f(z)-S\left[z f(z)-i_{\mathscr{R}}^{*} z f(z)\right]=S i_{\mathscr{R}}^{*} z f(z) .
\end{aligned}
$$

More generally, $S \mathscr{R}$ is contained in the range of $1-T T^{*}:$ Let $g(z)$ be in $\mathscr{R}$ such that $g(z)$ is orthogonal to $i_{\mathscr{R}}^{*} z f(z)$ for every $f(z)$ in $\mathscr{H}$. Then

$$
0=\left\langle g(z), i_{\mathscr{R}}^{*} z f(z)\right\rangle_{\mathscr{R}}=\langle g(z), z f(z)\rangle_{\mathscr{H}^{\prime}}=\langle S g(z), f(z)\rangle_{\mathscr{H}}
$$

for every $f(z)$ in $\mathscr{H}$. Letting $f(z)=S g(z)$, we conclude that $g(z)$ is constant. Hence $S \mathscr{R}=S \vee\left\{i_{\mathscr{R}}^{*} z f(z): f(z) \in \mathscr{H}\right\}$, which is contained in $\left(1-T T^{*}\right) \mathscr{H}$ since the rank of $1-T T^{*}$ is finite by the hypothesis.

It follows that $\mathscr{R}$ is finite dimensional since

$$
\operatorname{dim} \mathscr{R} \leq \operatorname{dim} S \mathscr{R}+\operatorname{dim} \operatorname{ker} S \leq \operatorname{rank}\left(1-T T^{*}\right)+\operatorname{dim} \mathscr{C} .
$$

Thus by the lemma $\mathscr{R}=\mathscr{H}^{\prime} \ominus \mathscr{F}$.

Furthermore, since $\mathscr{H}^{\prime}$ contains $\mathscr{C}$, the kernel of the restriction of $S$ to $\mathscr{H}^{\prime} \ominus \mathscr{F}$ is $\mathscr{C} \ominus\{f(0): f(z) \in \mathscr{F}\}$. Hence, we have that

$$
\begin{aligned}
\operatorname{dim} \mathscr{R} & =\operatorname{dim}[\mathscr{C} \ominus\{f(0): f(z) \in \mathscr{F}\}]+\operatorname{dim} S \mathscr{R} \\
& \leq \operatorname{dim} \mathscr{C}-\operatorname{dim}\{f(0): f(z) \in \mathscr{F}\}+\operatorname{rank}\left(1-T T^{*}\right) \\
& \leq \operatorname{dim} \mathscr{C}
\end{aligned}
$$

by the hypothesis. Therefore, $\mathscr{H}$ is isometrically equal to a space $\mathscr{H}(B)$.

Finally, any space which satisfies $(1)$ is at least a reducing subspace of $R(0)$ on some space $\mathscr{H}(B)$.

Corollary 3. Let $\mathscr{H}, \mathscr{F}$ and $T$ be defined as in Theorem 2 , but assume on the other hand that

$$
\delta=\operatorname{rank}\left(1-T T^{*}\right)-\operatorname{dim}\{f(0): f(z) \in \mathscr{F}\}
$$

is finite and positive. If $\tilde{\mathscr{C}}$ is any Hilbert space with dimension at least $\delta$, then $\mathscr{H} \oplus \tilde{\mathscr{C}}(z)$ is isometrically equal to a space $\mathscr{H}(B)$.

\section{ACKNOWLEDGMENT}

I am grateful to the referee for suggesting many improvements in this article.

\section{REFERENCES}

1. J. A. Ball and N. Cohen, De Branges-Rovnyak operator models and systems theory: A survey, Operator Theory: Advances and Applications, Vol. 50, Birkhäuser, Basel-Boston, 1991, pp. 93-136.

2. J. A. Ball and T. L. Kriete III, Operator-valued Nevanlinna-Pick kernels and the functional models for contraction operators, Integral Equations Operator Theory 10 (1987), 17-61.

3. A. Beurling, On two problems concerning linear transformations in Hilbert space, Acta Math. 81 (1949), 239-255.

4. L. de Branges, Factorization and invariant subspaces, J. Math. Anal. Appl. 29 (1970), 163-200. 
5. __ Krein spaces of analytic functions, J. Funct. Anal. 81 (1988), 219-259.

6. __ Square summable power series, Springer-Verlag (in preparation).

7. L. de Branges and J. Rovnyak, Appendix on square summable power series, canonical models in quantum scattering theory, Perturbation Theory and its Applications in Quantum Mechanics, Wiley, New York, 1966, pp. 347-392.

8. __ Square summable power series, Holt, Rinehart and Winston, New York, 1966.

9. J. Guyker, The de Branges-Rovnyak model, Proc. Amer. Math. Soc. 111 (1991), 95-99.

10. P. R. Halmos, Shifts on Hilbert spaces, J. Reine Angew. Math. 208 (1961), 102-112.

11. P. D. Lax, Translation invariant spaces, Acta Math. 101 (1959), 163-178.

12. N. K. Nikol'skii and V. I. Vasyunin, Notes on two function models, The Bieberbach Conjecture: Proc. Sympos. on the Occasion of the Proof, Math. Surveys Monographs, vol. 21, Amer. Math. Soc., Providence, RI, 1986, pp. 113-141.

13. J. Rovnyak, Ideals of square summable power series, Math. Mag. 33 (1960), 265-270; ibid. 34 (1961), 41-42.

14. __ Ideals of square summable power series, Proc. Amer. Math. Soc. 13 (1962), 360-365.

15. __ Ideals of square summable power series. II, Proc. Amer. Math. Soc. 16 (1965), 209-212.

16. D. Sarason, Doubly shift-invariant spaces in $H^{2}$, J. Operator Theory 16 (1986), 75-97.

17. __ Shift-invariant spaces from the Brangesian point of view, The Bieberbach Conjecture: Proc. Sympos. on the Occasion of the Proof, Math. Surveys Monographs, vol. 21, Amer. Math. Soc., Providence, RI, 1986, pp. 153-166.

Department of Mathematics, State University College of New York at Buffalo, Buffalo, New YorK 14222-1095

E-mail address: guykerj@snybufva 